\title{
Trans-thoracic Aortic Peak Velocity Variation for Goal Directed Fluid and Hemodynamic Therapy in Children and Postoperative Outcome: A Multicentric Randomized Controlled Trial: Editorial
}

\section{Claudine Kumba*}

Department of Pediatric Anesthesia and Critical Care, Necker Enfants Malades, University Hospital, Assistance Publique Hôpitaux de Paris, APHP, University of Paris, Paris, France

*Corresponding Author: Claudine Kumba, Department of Pediatric Anesthesia and Critical Care, Necker Enfants Malades, University Hospital, Assistance Publique Hôpitaux de Paris, APHP, University of Paris, Paris, France.
Received: March 06, 2020

Published: April 03, 2020

(C) All rights are reserved by Claudine

Kumba.

\section{Abstract}

A multicentric randomized controlled trial (RCT) is in preparation. This RCT has the primary objective to determine the impact of trans-thoracic aortic peak velocity variation in goal directed fluid and hemodynamic therapy (GDFHT) on postoperative morbidity in children in major surgery. This Editorial highlights essential points of this RCT in preparation.

Keywords: Children; Outcome; Goal Directed Fluid Hemodynamic Therapy

This RCT in preparation will be multicentric and will include 400 children aged less than 18 years in major surgery or major interventional procedures [1].

Why is it important to realize this study? As precised in earlier publications, postoperative outcome is multifactorial [2-5]. Among these multiple risk factors, patient's general state (ASA score, American Society of Anesthesiologists score) and surgery were identified as independent predictors [2-5].

The hypothesis is by optimizing patients fluid and hemodynamic state in major surgery intraoperatively, postoperative outcome in terms of morbidity defined as organ dysfunction will be improved. The aim of GDFHT is to optimize tissular perfusion. Tissular perfusion depends among others on oxygen delivery. The latter depends among others on cardiac output (CO).

Optimizing CO with GDFHT is an essential issue since hypovolemia and hypervolemia can lead to organ dysfunction.

What is new in this study? GDFHT has proven postoperative outcome improvement in adults [6]. In children, evidence of impact of intraoperative GDFHT on postoperative outcome is lacking. Aortic peak velocity variation has been validated for fluid responsiveness in children [7-9]. What has not been evidenced is the impact of this echocardiographic indice in GDFHT on postoperative outcome in children.
What is the primary objective of this RCT?

This multicentric RCT has primary objective to demonstrate that in children in major surgery or major interventional procedures, GDFHT with trans-thoracic aortic peak velocity variation improves postoperative morbidity in terms of organ dysfunction compared to control. Organ dysfunction will be defined according to the system or organ with clinical, biological and imaging findings.

The control group will be patients managed intraoperatively without aortic peak velocity variation to guide fluid and hemodynamic therapy.

What are the expected benefits of this RCT? Postoperative morbidity has been demonstrated to increase length of postoperative hospital stay (LOS). LOS has been shown to increase postoperative complications [10]. Reducing postoperative morbidity will decrease LOS. Increased LOS is related to high hospital costs.

What are the limits of this study?

Patients echogenicity, limited access to the chest and apical region and the familiarity of the medical team with echocardiographic technique can be limiting factors [11].

What is the strength of this trial? The multicentric randomized controlled aspect, large sample, non-invasive technique and innovation are strong points of this study $[12,13]$. 


\section{Bibliography}

1. Kumba C., et al. "Goal Directed Fluid and Hemodynamic Therapy and Postoperative Outcomes in Children: Value of TransThoracic Echocardiographic Aortic Blood Flow Peak Velocity Variation: A Multicentre Randomized Controlled Trial Protocol". Advances in Pediatric Research 7 (2020): 35.

2. Kumba C., et al. "Transfusion and Morbi-Mortality Factors: An Observational Descriptive Retrospective Pediatric Cohort Study". Journal of Anesthesia and Critical Care: Open Access 8.4 (2017): 00315.

3. Kumba C., et al. "Transfusion and Postoperative Out-come in Pediatric Abdominal Surgery". Journal of Clinical Research in Anesthesiology 1.1 (2018): 1-8.

4. Kumba C., et al. "Is Transfusion an Independent Risk Factor of Postoperative Outcome in Pediatric Orthopedic Surgical Patients? A Retrospective Study". Journal of Emergency Medicine and Critical Care 4.2 (2018): 7.

5. Kumba C., et al. "Blood Product Transfusion and Postoperative Outcome in Pediatric Neurosurgical Patients". EC Anaesthesia 4.8 (2018): 288-298.

6. Chong M., et al. "Does doal-directed haemodynamic and fluid therapy improve peri-operative outcomes ? A systématique review and meta-analysis". European Journal of Anaesthesiology 35.7 (2018): 469-483.

7. Desgranges FP., et al. "Respiratory Variation in Aortic Blood Flow Peak Velocity to Predict Fluid Responsivenes in Mechanically Ventilated Children: A Sytematic Review and Meta-Analysis". Pediatric Anesthesia 26 (2016): 37-47.

8. Morparia KG., et al. "Respiratory Variation in Peak Aortic Accurately Predicts Fluid Responsiveness in Children Undergoing Neurosurgery Under General Anesthesia”. Journal of Clinical Monitoring and Computing 32 (2018): 221-226.

9. Pereira de Souza Neto E., et al. "Predicting Fluid Responsiveness in Mechanically Ventilated Children Under General Anaesthesia Using Dynamic Parameters and Transthoracic Echocardiography". British Journal of Anaesthesia 106.6 (2018): 856-864.

10. Kumba C. "A Retrospective Descriptive Cohort Study of Preoperative, Intraoperative and Postoperative Management of Children in Scoliosis Surgery". EC Anaesthesia 5.2 (2019): 20-29.

11. Claudine Kumba. "Feasibility of Intraoperative Trans-Thoracic Echocardiography for Goal Directed Therapy in Children and Postoperative Outcome". Journal of Neonatal Research and Pediatrics Care 3.1 (2020): 180025.
12. Claudine Kumba. "Advances in Pediatric and Neonatal Research: Impact of Networking and Collaborating". EC Paediatrics 9.3 (2020): 01-02.

13. Claudine Kumba. "Innovating Applications with Trans-Thoracic Echocardiography in Goal Directed Fluid and Hemodynamic Therapy in Children". EC Clinical and Medical Case Reports 3.3 (2020): 01-03.

\section{Assets from publication with us}

- Prompt Acknowledgement after receiving the article

- Thorough Double blinded peer review

- Rapid Publication

- Issue of Publication Certificate

- High visibility of your Published work Website: www.actascientific.com/ Submit Article: www.actascientific.com/submission.php Email us: editor@actascientific.com Contact us: +919182824667 